\title{
SARS-CoV-2; Gestione di una postazione tampone
}

\author{
Duke Ashong $^{1}$ and Vera Pedone ${ }^{2}$ \\ ${ }^{1}$ University of Padua \\ ${ }^{2}$ AUSL Romagna
}

May 3, 2021

\section{Introduzione}

Alla luce della recente situazione di emergenza sanitaria di sanità pubblica come dichiarato dall'OMS il 30 gennaio 2020(Statement on the second meeting of the International Health Regulations (2005) Emergency Committee regarding the outbreak of novel coronavirus (2019-nCoV), n.d.) in merito alla diffusione dei casi di SARS-CoV-2 più comunemente noto come Coronavirus, considerato la notevole rilevanza sociale e contagiosità della patologia Covid-19, si è reso indispensabile visto l'eziopatogenesi, l'andamento geografico e temporale fornire ai professionisti sanitari tutti gli strumenti procedurali per fronteggiare al meglio questa sfida sanitaria.

Gli operatori sanitari, primi i colleghi cinesi e successivamente molti altri a livello internazionale si sono trovati in prima linea, quasi inconsapevolmente in prima battuta ad affrontare casi di sospetti quadri clinici con interessamento delle vie respiratorie senza i dispositivi di protezione individuale adeguate. Questo breve articolo vuole fornire indicazioni chiare ed esemplificative attraverso la creazione di una procedura ad hoc, per consentire :

- L'autoprotezione dei professionisti sanitari durante il prelievo di materiale biologico indispensabile per la diagnosi di Covid-19.

- La riduzione della trasmissione dell'infezioni da sanitari ad altri pazienti e a tutta la popolazione generale

Con il fine prioritario di tutelare la salute del professionista sanitario e indirettamente della popolazione generale.

I coronavirus umani si trasmettono da una persona infetta principalmente per via aerea e tramite contatti diretti. La sintomatologia include:Cefalea, rinorrea, tosse, faringite, piressia e una sensazione generale di malessere tra le complicanze vi sono la polmonite e bronchite in modo marcato nelle persone con comorbidità a carico del apparato cardiocircolatorio e del tratto respiratorio (Coronavirus - Sintomi e diagnosi, n.d.). I sintomi sono simili all'influenza, aspecifici, pertanto la diagnosi laboratoriale(DIAGNOSTICA DI LABORATORIO DELLA SINDROME RESPIRATORIA ACUTA (SARS), n.d.) e strumentale(COVID-19: il ruolo della TAC nella diagnosi - Humanitas News, n.d.) è indispensabile al fine di trattare al meglio i pazienti critici in strutture sanitarie e collocare i pazienti paucisintomatici/asintomatici in isolamento.

\section{GUIDA}

\section{EFFETTUAZIONE DEL TAMPONE RINO-FARINGEO PER SARS-CoV-2}

Ove possibile eseguire l'attività "pre-tampone" prima di arrivare nella zona tampone al fine di ridurre la possibilità di contaminazione.

\section{Attività Pre-tampone}


Il personale sanitario:

- esegue l'accettazione telefonicamente compilando specifico foglio di notifica con relativa etichetta

- raccomanda all'utente, ove possibile, di presentarsi a digiuno, senza aver lavato i denti e senza l'uso di colluttori orali( Tampone (Istituto superiore di sanità), n.d.)

- raccomanda al paziente di presentarsi nel posto e all'orario stabilito munito di mascherina chirurgica, con mezzo di trasporto proprio e preferibilmente senza accompagnatori.

- controlla la checklist tampone controllando la presenza di tutti i materiali necessari per la buona riuscita del prelievo del campione biologico 


\begin{tabular}{|c|c|}
\hline \multicolumn{2}{|l|}{ CHECKLIST MATERIALE TAMPONE } \\
\hline Abbassalingua & \\
\hline Abito da lavoro manica lunga & \\
\hline Alibox & \\
\hline Busta con DPI puliti di scorta & \\
\hline Busta per DPI precedentemente decontaminati riutilizzabili (occhiali protettivi) & \\
\hline Calzari & \\
\hline Carta per pulizia superfici & \\
\hline Cerotto di carta & \\
\hline Contenitore rifiuti rischio biologico & \\
\hline Contenitore secondario a tenuta per trasporto provette & \\
\hline Copricapo lungo bianco & \\
\hline Cuffia capelli & \\
\hline Disinfettante per superfici & \\
\hline Etichette & \\
\hline Filtrante repiratoriio FFP2/FFP3 & \\
\hline Fogli di notifica & \\
\hline Forbice & \\
\hline Gel alcolico con erogatore & \\
\hline Guanti in nitrile e chirurgici & \\
\hline Lista utenti in raccoglitore plastificato & \\
\hline Mascherine chirugiche & \\
\hline Provette tamponi & \\
\hline Sacchetto a doppia tasca per campioni biologici & \\
\hline Sacchetti di plastica & \\
\hline Sovracamice monuso idrorepellente/Tuta monuso & \\
\hline Schermo facciale e/o Occhiali Protettivi & \\
\hline
\end{tabular}

\section{Firma Operatore}




\section{PREDISPOSIZIONE DEL MATERIALE}

Una volta in sede, delinea la "ZONA PULITA" e la "ZONA SPORCA". Tutti gli altri DPI durante l'esecuzione del tampone, dovranno rimanere all'interno della ZONA PULITA ed in caso di necessità, potranno essere prelevati dal primo operatore li passerà al secondo operatore, il quale durante l'esecuzione dei tamponi dovrà rimanere nella ZONA SPORCA.

\section{- FASE DELLA VESTIZIONE}

\section{ESECUZIONE DEL TAMPONE}

Il personale sanitario presente nella ZONA PULITA nella fase di preparazione:

- effettua l'igiene delle mani

- reperisce il tampone sterile specifico per l'indagine dalla ZONA PULITA

- procede all'identificazione dell'utente e alla registrazione dei dati anagrafici

- consegna la provetta etichettata con i dati dell'utente, al secondo operatore che eseguirà il tampone

Il personale sanitario presente nella ZONA SPORCA:

- spiega al paziente le fasi e l'utilità della manovra che si sta per eseguire affinché egli comprenda pienamente ciò che verrà effettuato e aumenti la sua collaborazione

- avvisa il paziente che potrebbe avere dei conati di vomito durante l'esecuzione della manovra e lo istruisce a pronunciare la lettera "A", poiché in questo modo si attenua il riflesso del vomito. Avvertirlo che potrebbe avere un po' di fastidio.

\section{Tampone Oro-faringeo(Tamponi e assistenza infermieristica, n.d.)}

Il personale sanitario:

- effettua l'igiene delle mani ;

- invita il paziente ad inclinare la testa all'indietro e ad aprire bene la bocca ;

- estrae il tampone dalla custodia ;

- in caso di necessità utilizza un abbassalingua sterile per facilitare il prelievo;

- inserisce il tampone dietro l'ugola e tra i pilastri tonsillari, evitando il contatto con altre zone dell'interno della bocca;

- strofina il tampone soprattutto nelle zone con segno di flogosi evitando contaminazioni con la saliva.

\section{Tampone nasale}

Il personale sanitario:

- invita il paziente a tenere il busto eretto e ad inclinare leggermente la testa all'indietro;

- estrae il tampone dalla custodia

- inserisce il tampone nella narice e prosegue lungo il pavimento della coana nasale fino a raggiungere il retro del rinofaringe

- ruota delicatamente il tampone e lo mantiene in sitù per qualche secondo al fine di raccogliere abbondante secreto nasale

In caso di difficoltà ripete la manovra nell'altra narice.

Il secondo operatore nella fase successiva all'esecuzione del test: 
- Posiziona il foglio di notifica/documento di accompagnamento nel sacchetto a doppia tasca per campioni biologici

- Porge successivamente il sacchetto con la tasca chiudibile, aperta in modo tale da far scivolare la provetta all'interno dal secondo operatore senza toccarne i bordi

- Ripone il sacchetto con il tampone nel frigorifero ad una temperatura di $4^{\circ}$ gradi.

Alla fine della procedura entrambi gli operatori eseguiranno un frizionamento alcolico delle mani, il secondo operatore dovrà prima togliere il secondo paio di guanti. Indossandone un altro prima di fare il tampone successivo.

\section{- FASE DELLA SVESTIZIONE.}

- Trasposto del materiale biologico:Confezionare il pacco per i campioni clinici utilizzando contenitori adeguati a norma di legge (Circolare n. 3 dell'8 maggio 2003 del Ministero della Salute) per l'invio dio materiale biologico,categoria B codice UN3373. E' fatto obbligo di utilizzare un triplo imballaggio, formato da un imballo esterno, uno intermedio ed uno interno a tenuta stagna, conforme alle disposizioni vigenti.I campioni vanno mantenuti refrigerati $\left(+4-8^{\circ} \mathrm{C}\right)$ prima dell'invio(Istruzioni operative per l'invio di campioni relativi alla diagnosi di infezione da Coronavirus emergenti (MERS-CoV, SARS, 2019-nCoV), n.d.).

Conclusione

L'attività di esecuzione del tampone oro-faringeo può essere eseguita da professionisti sanitari tra cui

- Assistente sanitario, inquadrato secondo il D. M 17 gennaio 1997 n. 69 come professionista sanitario operante nell'ambito della prevenzione primaria, secondaria e terziaria, della promozione della salute, dell'educazione alla salute individuale, familiare, di gruppo, di collettività ed istituzionale, della ricerca epidemiologica e sanitario-sociale, della medicina sociale, dell'igiene, della sanità pubblica, dell'assistenza sanitaria e socio -sanitaria integrata attraverso le tecniche di monitoraggio della salute, le tecniche di monitoraggio biologico applicate alle malattie infettive, alla medicina del lavoro, legale, sociale, mentale e delle dipendenze(Esercizio dell'attività professionale Assistente sanitario, n.d.).

- Infermiere, inquadrato secondo il D. M 739/1994 n. 739 come addetto all'assistenza infermieristica preventiva, curativa, palliativa e riabilitativa è di natura tecnica, relazionale, educativa. Le principali funzioni sono la prevenzione delle malattie, l'assistenza dei malati e dei disabili di tutte le età e l'educazione sanitaria (Profilo prof. Infermiere, n.d.)

- Medico chirurgo

Questa attività oltre ai fini diagnostici nei confronti del singolo paziente,risulta fondamentale in quanto permette di monitorare l'andamento geografico e temporale della diffusione da SARS-CoV2

\section{Definizione}

Attivita Pretampone: tutte le attività che si possono eseguire prima di arrivare nella zona addebita ai tamponi Checklist tampone: Tutto làoccorrente per eseguire in sicurezza la raccolta di materiale biologico

Primo operatore: operatore assiste il secondo operatore

Secondo operatore: operatore preposto all'esecuzione del tampne

Zona pulita: spazio dedicato alle azioni del primo operatore, il quale non ha contatti diretti con i pazienti da tamponare. 
Zona sporca: spazio dedicato alle azioni del secondo operatore, il quale ha contatti diretti con i pazienti da tamponare

Zona tampone: zona allestita ed addebita al prelievo di materiale biologico (Tampone)

\section{References}

https://www . who. int/news-room/detail/30-01-2020-statement-on-the-second-meeting-

of-the-international-health-regulations-(2005)-emergency-committee-regarding-the-

outbreak-of-novel-coronavirus-(2019-ncov). https://www.who.int/news-room/detail/30-01-

2020-statement-on-the-second-meeting-of-the-international-health-regulations-(2005)-

emergency-committee-regarding-the-outbreak-of-novel-coronavirus-(2019-ncov)

https://www.epicentro.iss.it/coronavirus/sintomi-diagnosi. https://www.epicentro.iss.it/ coronavirus/sintomi-diagnosi

http://www.trapianti.salute.gov.it/resources/static/primopiano/sars2506/diagnostica.pdf . http://www.trapianti.salute.gov.it/resources/static/primopiano/sars2506/diagnostica.pdf

https://www.humanitas.it/news/25737-covid-19-ruolo-della-tac-nella-diagnosi. https://www . humanitas.it/news/25737-covid-19-ruolo-della-tac-nella-diagnosi

https://www.issalute.it/index.php/la-salute-dalla-a-alla-z-menu/t/tampone-analisicliniche\#il-test. https://www.issalute.it/index.php/la-salute-dalla-a-alla-z-menu/t/ tampone-analisi-cliniche\#il-test

https://www.nurse24.it/studenti/indagini-diagnostiche/raccolta-di-campionimicrobiologici-mediante-tampone.html. http://www.nurse24.it/studenti/indaginidiagnostiche/raccolta-di-campioni-microbiologici-mediante-tampone.html

https://www.inmi.it/wp-content/uploads/2020/01/Allegato-1_Istruzioni-invio-campioni.pdf . https ://www.inmi.it/wp-content/uploads/2020/01/Allegato-1_Istruzioni-invio-campioni.pdf

https://ape.agenas.it/documenti/provider/CodiceDeontologico_AssistenteSanitario.pdf . https://ape.agenas.it/documenti/provider/CodiceDeontologico_AssistenteSanitario.pdf

http://www.salute.gov.it/imgs/C_17_normativa_1870_allegato.pdf. http://www.salute.gov.it/ imgs/C_17_normativa_1870_allegato.pdf 